\title{
The Degree of Approximation and Converse Theorems with Exponential-Type Weights
}

\author{
Ryozi SAKAI \\ Department of Mathematics, Meijo University, Tenpaku-ku Nagoya 468-8502, Japan \\ Email: ryozi@crest.ocn.ne.jp
}

\begin{abstract}
Let $\mathbb{R}=(-\infty, \infty)$, and let $Q \in \mathbf{C}^{\mathbf{1}}(\mathbb{R}): \mathbb{R} \rightarrow[0, \infty)$ be an even function, which is an exponent. We deal with the exponential-type weights $w(x)=e^{-Q(x)}, \quad x \in \mathbb{R}$. In this paper, we consider the approximation problem with the weight $w(x)$, and then we give some converse theorems, and investigate the smoothness of functions. We will also study the connections of the degree of approximation of a function between different norms. To do them we need to give the Nikolskii-type inequalities.
\end{abstract}

MSC; 41A17

Keywords: exponential-type weights, degree of approximation, Nikolskii-type inequalities

\section{Introduction and Theorems}

Let $\mathbb{R}=(-\infty, \infty)$, and let $Q \in \mathbf{C}^{\mathbf{1}}(\mathbb{R}): \mathbb{R} \rightarrow[0, \infty)$ be an even function. We consider the weights $w(x):=\exp (-Q(x))$ satisfying $\int_{0}^{\infty} x^{n} w^{2}(x) d x<\infty$ for all $n=0,1,2, \ldots$

Mhaskar [1] investigates the smoothness of functions, and gives some converse theorems. If $f: \mathbb{R} \rightarrow \mathbb{R}$ is measurable, we define

$$
\|f w\|_{L_{p}(\mathbb{R})}:= \begin{cases}\left(\int_{-\infty}^{\infty}|f(t) w(t)|^{p} d t\right)^{1 / p}, & \text { if } 0<p<\infty \\ \sup _{t \in \mathbb{R}}|f(t) w(t)|, & \text { if } p=\infty,\end{cases}
$$

where if $p=\infty$, we suppose that $f$ is continuous on $\mathbb{R}$, and $\lim _{|x| \rightarrow \infty} w(x) f(x)=0$, then we write $w f \in C_{0}(\mathbb{R})$. The class of all functions $f$ for which $\|w f\|_{L_{p}(\mathbb{R})}<\infty$ will be denoted by $L_{p, w}(\mathbb{R})$, with the usual understanding that two functions are identified if they are equal almost everywhere. For $f \in L_{p, w}(\mathbb{R})(1 \leqslant p \leqslant \infty)$ the degree of weighted polynomial approximation is defined by

$$
E_{p, n}(w ; f):=\inf _{P \in \mathcal{P}_{n}}\|w(f-P)\|_{L_{p}(\mathbb{R})},
$$

where $\mathcal{P}_{n}$ denotes the class of all polynomials $P_{n}$ with degree $\leqslant n$. Let $Q(x)=\log w(x)^{-1}$ be an even and convex function on $\mathbb{R}$, and let $Q$ be continuously differentiable on $(0, \infty)$. Furthermore, there are constants $c_{1}$ and $c_{2}$ such that

$$
0<c_{1} \leqslant \frac{x Q^{\prime}(x)}{Q(x)} \leqslant c_{2}<\infty
$$

for all $x \in(0, \infty)$. Then we say that $w=\exp (-Q(x))$ is a Freud weight. We define $q_{x}$ by $q_{x} Q^{\prime}\left(q_{x}\right)=x$. For $f \in C^{s}(\mathbb{R})$ Mhaskar [1] gives a direct theorem as follows:

$$
E_{p, n}(w ; f) \leqslant C\left(\frac{q_{n}}{n}\right)^{s} K_{r, p}\left(f^{(s)} ; \frac{q_{n}}{n}\right) .
$$

Here $K_{r, p}(f ; \delta), \delta>0$ is the $\mathrm{K}$-functional which is defined by

$$
K_{r, p}(f ; \delta):=\inf \left\{\|w(f-g)\|_{L_{p}(\mathbb{R})}+\delta^{r}\left\|w g^{(r)}\right\|_{L_{p}(\mathbb{R})}\right\},
$$

where the infimum is over all function $g$ having an absolutely continuous $(r-1)$-st order derivatives and $w g^{(r)} \in L_{p}(\mathbb{R})$. Furthermore, he also gives the following the inverse theorem. 
Mhaskar's Theorem ([1]). Let $Q^{\prime \prime}$ be increasing on $(0, \infty)$, let $s \geqslant 0$ be an integer, and let $1 \leqslant p, q \leqslant \infty$. (i) Let $p \leqslant q, f \in L_{q, w}(\mathbb{R})$. If

$$
\sum_{n=1}^{\infty} q_{n}^{\frac{1}{p}-\frac{1}{q}-s} n^{s-1} E_{q, n}(w ; f)<\infty
$$

then $f$ is an $s$-times iterated integral of a function $f^{(s)} \in L_{p, w}(\mathbb{R})$, and for the integer $n \geqslant s$,

$$
E_{p, n-s}\left(w ; f^{(s)}\right) \leqslant c \sum_{k=1}^{\infty} q_{k n}^{\frac{1}{p}-\frac{1}{q}-s}(k n)^{s} \frac{E_{q, k n}(w ; f)}{k} .
$$

(ii) Let $q \leqslant p$, and let $f \in L_{q, w}(\mathbb{R})$. If

$$
\sum_{n=1}^{\infty}\left(\frac{n}{q_{n}}\right)^{\frac{1}{q}-\frac{1}{p}-s} \frac{E_{q, n}(w ; f)}{n}<\infty,
$$

then $f$ is an $s$-times iterated integral of a function $f^{(s)} \in L_{p, w}(\mathbb{R})$, and for the integer $n \geqslant s$,

$$
E_{p, n-s}\left(w ; f^{(s)}\right) \leqslant c \sum_{k=1}^{\infty}\left(\frac{k n}{q_{k n}}\right)^{\frac{1}{q}-\frac{1}{p}-s} \frac{E_{q, k n}(w ; f)}{k} .
$$

(iii) Let $f \in L_{q, w}(\mathbb{R})$. If

$$
\sum_{n=1}^{\infty}\left(\frac{n}{q_{n}}\right)^{\left|\frac{1}{q}-\frac{1}{p}\right|-s} \frac{E_{q, n}(w ; f)}{n}<\infty
$$

then $f$ is an $s$-times iterated integral of a function $f^{(s)} \in L_{p, w}(\mathbb{R})$, and for the integer $n \geqslant s$,

$$
E_{p, n-s}\left(w ; f^{(s)}\right) \leqslant c \sum_{k=1}^{\infty}\left(\frac{k n}{q_{k n}}\right)^{\left|\frac{1}{q}-\frac{1}{p}\right|-s} \frac{E_{q, k n}(w ; f)}{k} .
$$

For a long time such problems have been studied, for example, we find some results in [2]. In recently years we can find $[3,4]$ ).

In this paper, we will give some analogies of Mhaskar's results with the Freud weights, and extend to the results with Erdös-type weights. We give some converse theorems, and investigate the smoothness of functions. We will also study the connections of the degrees of approximation of a function between different norms. To prove them we will follow Mhaskar's methods.

In Section 2 we give theorems and some preliminaries. In Section 3 we write some lemmas, and prove theorems. In Section 4 we prove Corollaries 2.10 and 2.11 .

Throughout this paper $C, C_{1}, C_{2}, \ldots$ denote positive constants independent of $n, x, t$ or polynomials $P(x)$. The same symbol does not necessarily denote the same constant in different occurrences. Let $\mathcal{P}_{n}$ be the class of all polynomials with degree $n$ at most.

\section{Theorems and Preliminaries}

First we start the following definition from [5]. We say that $f: \mathbb{R} \rightarrow[0, \infty)$ is quasi-increasing if there exists $C>0$ such that $f(x) \leqslant C f(y), 0<x<y$.

Definition 2.1. We define $w=\exp (-Q) \in \mathcal{F}\left(C^{2}+\right)$ as follows: Let $Q: \mathbb{R} \rightarrow[0, \infty)$ be a continuous even function, and satisfy the following properties:

(a) $Q^{\prime}(x)$ is continuous in $\mathbb{R}$, with $Q(0)=0$.

(b) $Q^{\prime \prime}(x)$ exists and is positive in $\mathbb{R} \backslash\{0\}$.

(c) $\lim _{x \rightarrow \infty} Q(x)=\infty$. 
(d) The function

$$
T(x):=\frac{x Q^{\prime}(x)}{Q(x)}, \quad x \neq 0
$$

is quasi-increasing in $(0, \infty)$, with

$$
T(x) \geqslant \Lambda>1, \quad x \in \mathbb{R} \backslash\{0\} .
$$

(e) There exists $C_{1}>0$ such that

$$
\frac{Q^{\prime \prime}(x)}{\left|Q^{\prime}(x)\right|} \leqslant C_{1} \frac{\left|Q^{\prime}(x)\right|}{Q(x)}, \text { a.e. } x \in \mathbb{R} \backslash\{0\}
$$

and there also exists a compact subinterval $J(\ni 0)$ of $\mathbb{R}$, and $C_{2}>0$ such that

$$
\frac{Q^{\prime \prime}(x)}{\left|Q^{\prime}(x)\right|} \geqslant C_{2} \frac{\left|Q^{\prime}(x)\right|}{Q(x)}, \text { a.e. } x \in \mathbb{R} \backslash J .
$$

Example 2.2. (i) If $T(x)$ is bounded, then we call the weight $w=\exp (-Q)$ the Freud-type weight. The following example is the Freud-type weight.

$$
w(x)=\exp \left(-|x|^{\gamma}\right), \quad \gamma>1
$$

If $T(x)$ is unbounded, then we call the weight $w=\exp (-Q)$ the Erdös-type weight. The following example give the Erdös-type weight $w=\exp (-Q)$.

(ii) $([5,6])$. For $\gamma>1, l=1,2,3, \ldots$.

$$
Q(x)=Q_{l ; \gamma}(x)=\exp _{l}\left(|x|^{\gamma}\right)-\exp _{l}(0)
$$

where

$$
\exp _{l}(x)=\exp (\exp (\exp \ldots \exp x) \ldots) \quad(l \text {-times })
$$

More generally, we define for $\gamma+u>1, \gamma \geqslant 0, u \geqslant 0$ and $l \geqslant 1$,

$$
Q_{l ; \gamma, u}(x):=|x|^{u}\left(\exp _{l}\left(|x|^{\gamma}\right)-\gamma^{*} \exp _{l}(0)\right)
$$

where $\gamma^{*}=0$ if $\gamma=0$, otherwise $\gamma^{*}=1$. We note that $Q_{l ; 0, u}$ gives a Freud-type weight.

(iii) We define $Q_{\gamma}(x):=(1+|x|)^{|x|^{\gamma}}-1, \quad \gamma>1$.

We need the following assumption:

Assumption 2.3. Let $w(x)=\exp (-Q(x)) \in \mathcal{F}\left(C^{2}+\right)$, and let $r \geqslant 1$ be an integer. Let the exponent $Q$ satisfy that for $|x| \geqslant K>0$ large enough, $Q \in C^{(r+2)}(\mathbb{R} \backslash\{0\})$ and

$$
\left|\frac{Q^{(r+2)}(x)}{Q^{(r+1)}(x)}\right| \leqslant C\left|\frac{Q^{(r+1)}(x)}{Q^{(r)}(x)}\right| \sim\left|\frac{Q^{(r)}(x)}{Q^{(r-1)}(x)}\right| \sim \ldots \sim\left|\frac{Q^{\prime}(x)}{Q(x)}\right|,
$$

and for some $0<\lambda<(r+2) /(r+1), C>0$, then for $|x| \geqslant K>0$ large enough,

$$
\frac{\left|Q^{\prime}(x)\right|}{Q(x)^{\lambda}} \leqslant C
$$

Then we write $w \in \mathcal{F}_{\lambda}\left(C^{r+2}+\right)$. 
Remark 2.4. (i) All in Example 2.2 satisfy all conditions of Assumption 2.3 for all $\gamma=1,2,3, \ldots$ or $\gamma \geqslant r$.

(ii) More generally, we can give the examples of weights $w \in \mathcal{F}_{\lambda}\left(C^{r+2}+\right)$. Let $w=\exp (-Q) \in \mathcal{F}\left(C^{2}+\right)$, and let us define

$$
\mu_{+}:=\limsup _{x \rightarrow \infty} \frac{Q^{\prime \prime}(x)}{Q^{\prime}(x)} / \frac{Q^{\prime}(x)}{Q(x)}, \quad \mu_{-}:=\liminf _{x \rightarrow \infty} \frac{Q^{\prime \prime}(x)}{Q^{\prime}(x)} / \frac{Q^{\prime}(x)}{Q(x)}
$$

If $\mu_{+}=\mu_{-}$, then we say that the weight $w$ is regular. If $Q \in \mathbf{C}^{(r+2)}(\mathbb{R} \backslash\{0\})$ satisfies (1), then for the regular weights we have $w=\exp (-Q) \in \mathcal{F}_{\lambda}\left(C^{r+2}+\right)$, that is, (2) holds (see [7]).

We need the Mhaskar-Rakhmanov-Saff numbers $a_{x}$;

$$
x=\frac{2}{\pi} \int_{0}^{1} \frac{a_{x} u Q^{\prime}\left(a_{x} u\right)}{\left(1-u^{2}\right)^{1 / 2}} d u, x>0 .
$$

The following theorems are important.

Theorem 2.5 ([7]). Let $0<\lambda<3 / 2$ and $\alpha \in \mathbb{R}$. Then for $w=\exp (-Q) \in \mathcal{F}_{\lambda}\left(C^{3}+\right)$, we can construct a new weight $w_{\alpha} \in \mathcal{F}\left(C^{2}+\right)$ such that

$$
T(x)^{\alpha} w(x) \sim w_{\alpha}(x), \quad x \in \mathbb{R},
$$

and the following holds

$$
a_{n}\left(w_{\alpha}\right) \sim a_{n}=a_{n}(w), \quad n=1,2,, 3 \ldots .
$$

In fact, there exists $c>1$ such that

$$
a_{n / c}\left(w_{\alpha}\right) \leqslant a_{n}=a_{n}(w) \leqslant a_{c n}\left(w_{\alpha}\right), \quad n=1,2,3, \ldots
$$

and

$$
T_{w_{\alpha}}(x) \sim T(x)=T_{w}(x) \quad x \in \mathbb{R} .
$$

Moreover, for $\alpha_{1}, \alpha_{2}, \ldots, \alpha_{k}, k \leqslant m$ we obtain an iterated weight $w_{\alpha_{1}, \alpha_{2}, \ldots, \alpha_{k}}$.

Theorem 2.6 (cf. [7]). Let the weight $w=\exp (-Q) \in \mathcal{F}_{\lambda}\left(C^{r+2}+\right)(0<\lambda<(r+2) /(r+1))$. Then for $\alpha_{k} \in \mathbb{R}, k=1,2, \ldots, r$, we can construct an iterated weight $w_{\left(\alpha_{1}, \ldots, \alpha_{k} ; k\right)}(x) \in \mathcal{F}\left(C^{2}+\right)$ such that

$$
T(x)^{\alpha_{1}+\ldots+\alpha_{k}} w(x) \sim T(x)^{\alpha_{k}} w_{\left(\alpha_{1}, \ldots, \alpha_{k-1}: k-1\right)}(x) \sim w_{\left(\alpha_{1}, \ldots, \alpha_{k} ; k\right)}(x),
$$

where $w_{\left(\alpha_{0}, 0\right)}(x)=w(x)$. Then we also have

$$
w_{\left(\alpha_{1}, \ldots, \alpha_{k}: k\right)}(x) \sim w_{\left(\alpha_{1}+\ldots+\alpha_{k} ; 1\right)}(x) .
$$

Proof. Using Theorem 2.5, we can inductively construct new weights $w_{i}=\exp \left(-Q_{i}\right) \in \mathcal{F}_{\lambda}\left(C^{r+2-i}\right)$ for $w=\exp (-Q) \in \mathcal{F}_{\lambda}\left(C^{r+2}+\right)$ as follows:

$$
\begin{aligned}
& w_{\left(\alpha_{1}+\ldots+\alpha_{k} ; 1\right)}(x) \sim T(x)^{\alpha_{1}+\ldots+\alpha_{k}} w(x) \sim T(x)^{\alpha_{k}} w_{\left(\alpha_{1}, \ldots, \alpha_{k-1} ; k-1\right)}(x) \\
& \sim w_{\left(\alpha_{1}, \ldots, \alpha_{k} ; k\right)}(x) .
\end{aligned}
$$

We omit the details (see [7]). \#

Remark 2.7. When $\alpha=\alpha_{1}=\alpha_{2}=\ldots=\alpha_{k}$, we write $w_{(\alpha ; k)} \sim w_{k \alpha ; 1)}=$ : $w_{k \alpha}$. If $\alpha \neq \beta$, then $T^{\beta} w_{\alpha} \sim\left(w_{\alpha}\right)_{\beta} \in \mathcal{F}\left(C^{2}+\right)$.

Now, we extend Mhaskar's theorem as follows. 
Theorem 2.8. Let $s \geqslant 1$ be an integer. We assume $a_{n} \leqslant C n^{1 / 2}$ if $T(x)$ is bounded.

(i) Let $w=\exp (-Q) \in \mathcal{F}_{\lambda}\left(C^{s+2}+\right)(0<\lambda<(s+2) /(s+1))$, and let $1 \leqslant p \leqslant q \leqslant \infty$. Let $\sqrt{T}{ }^{s} f \in L_{q, w}(\mathbb{R})$. If

$$
\sum_{k=1}^{\infty} a_{k}^{\frac{1}{p}-\frac{1}{q}-s} k^{s-1} E_{q, k}\left(T^{\frac{s}{2}} w ; f\right)<\infty
$$

then $f$ is an $s$-times iterated integral of functaion $f^{(s)} \in L_{p, w}(\mathbb{R})$, and for the integer $n \geqslant s$,

$$
E_{p, n-s}\left(w ; f^{(s)}\right) \leqslant C \sum_{k=1}^{\infty} a_{k n}^{\frac{1}{p}-\frac{1}{q}-s}(k n)^{s} \frac{E_{q, k n}\left(T^{\frac{s}{2}} w ; f\right)}{k} .
$$

(ii) Let $w=\exp (-Q) \in \mathcal{F}_{\lambda}\left(C^{s+3}+\right)(0<\lambda<(s+3) /(s+2)), 1 \leqslant p, q \leqslant \infty$, and let $\sqrt{T} s+\left|\frac{1}{q}-\frac{1}{p}\right| f \in$ $L_{q, w}(\mathbb{R})$. If

$$
\sum_{k=1}^{\infty}\left(\frac{k}{a_{k}}\right)^{\left|\frac{1}{q}-\frac{1}{p}\right|+s} \frac{E_{q, k}\left(T^{\left(s+\left|\frac{1}{q}-\frac{1}{p}\right|\right) / 2} w ; f\right)}{k}<\infty
$$

then $f$ is an $s$-times iterated integral of a function $f^{(s)} \in L_{p, w}(\mathbb{R})$, and for the integer $n \geqslant s$,

$$
E_{p, n-s}\left(w ; f^{(s)}\right) \leqslant C \sum_{k=1}^{\infty}\left(\frac{k n}{a_{k n}}\right)^{\left|\frac{1}{q}-\frac{1}{p}\right|+s} \frac{E_{q, k n}\left(T^{\left(s+\left|\frac{1}{q}-\frac{1}{p}\right|\right) / 2} w ; f\right)}{k} .
$$

Remark 2.9. (i) For a Freud-type weight we have $a_{n} \sim q_{n}$. In fact, from [1] and $\frac{Q^{\prime}\left(q_{2 n}\right)}{Q^{\prime}\left(q_{n}\right)}=\frac{2 q_{n}}{q_{2 n}}$ we conclude this result. Therefore, we may consider that Theorem 2.8 and Mhaskar's Theorem is equivalent. (ii) If $T(x)$ is unbounded, then for every $\eta>0$ we have $a_{n} \leqslant C(\eta) n^{\eta}$, where $C(\eta)$ is a constant depending only on $\eta$ (see [8]).

Corollary 2.10. (i) Let $s \geqslant 1$ be an integer, and let $1 \leqslant p \leqslant \infty$. Let $w=\exp (-Q) \in \mathcal{F}_{\lambda}\left(C^{s+2}+\right)(0<$ $\lambda<(s+2) /(s+1))$. Let $\sqrt{T}^{s} f \in L_{p, w}(\mathbb{R})$, and $\beta>s$. If

$$
E_{p, n}\left(w_{\left(\frac{1}{2} ; s\right)} ; f\right) \sim E_{p, n}\left(\sqrt{T}^{s} w ; f\right)=\mathcal{O}\left(\frac{a_{n}}{n}\right)^{\beta},
$$

then $f$ is an $s$-times iterated integral of a function $f^{(s)} \in L_{p, w}(\mathbb{R})$, and

$$
E_{p, n}\left(w ; f^{(s)}\right)=\mathcal{O}\left(\frac{a_{n}}{n}\right)^{\beta-s} .
$$

(ii) Let $s \geqslant 1$ be an integer, and let $1 \leqslant p \leqslant q \leqslant \infty$. Let $w=\exp (-Q) \in \mathcal{F}_{\lambda}\left(C^{s+3}+\right)(0<\lambda<$ $(s+3) /(s+2))$. We put $\alpha:=1 / p-1 / q$. If $\sqrt{T}^{s+\alpha} f \in L_{q, w}(\mathbb{R})$, and if for some $\beta>s+\alpha$,

$$
E_{q, n}\left(\left\{w_{\frac{\alpha}{2}}\right\}_{\left(\frac{1}{2} ; s\right)} ; f\right) \sim E_{q, n}\left(T^{(s+\alpha) / 2} w ; f\right)=\mathcal{O}\left(\frac{a_{n}}{n}\right)^{\beta}
$$

then $f$ is an $s$-times iterated integral of a function $f^{(s)} \in L_{p, w}(\mathbb{R})$, and

$$
E_{p, n}\left(w ; f^{(s)}\right)=\mathcal{O}\left(\frac{a_{n}}{n}\right)^{\beta-s-\alpha}
$$

Let $\gamma>1$, and let $l \geqslant 1$ be an integer. Then we set

$$
w_{l ; \gamma}(x):=\exp \left(-Q_{l ; \gamma}(x)\right), \quad Q_{l ; \gamma}(x):=\exp _{l}\left(|x|^{\gamma}\right)-\exp _{l}(0) .
$$

The following theorem is given for a specific weight $w_{l, \gamma}$. 
Corollary 2.11. Let $s$ be a nonnegative integer, and let $1 \leqslant p \leqslant q \leqslant \infty$. Let $\sqrt{T}^{s} f \in L_{q, w_{l ; \gamma}}$, $\beta>\frac{1}{p}-\frac{1}{q}+s$, and let $\delta$ be a fixed as $0<\delta<\beta-s$. If we suppose

$$
E_{q, n}\left(\left\{w_{l ; \gamma}\right\}_{\frac{s}{2}} ; f\right) \sim E_{q, n}\left(\sqrt{T}^{s} w_{l ; \gamma} ; f\right)=\mathcal{O}\left(\left(\frac{\left(\log _{l} n\right)^{\frac{1}{\gamma}}}{n}\right)^{\beta}\right),
$$

then $f$ is an $s$-times iterated integral of a function $f^{(s)} \in L_{p, w}(\mathbb{R})$, and for the integer $n \geqslant s, w_{l ; \gamma} f \in L_{p}(\mathbb{R})$,

$$
E_{p, n}\left(w_{l ; \gamma} ; f^{(s)}\right)=\left\{\begin{array}{l}
\mathcal{O}\left(n^{-\beta+s+\delta}\right), \quad \text { for } l=1 \text { and } \beta-s-\frac{1}{q}+\frac{1}{p}>\gamma \\
\mathcal{O}\left(n^{-\beta+s}\left(\log _{l} n\right)^{\frac{1}{\gamma}\left(\beta-s-\frac{1}{q}+\frac{1}{p}\right)}\right), \quad \text { otherwise. }
\end{array}\right.
$$

\section{Proof of Theorems}

To prove theorems we need some fundamental lemmas.

Lemma 3.1. (i) [5] For a fixed $L>0$ and uniformly for $t>0$,

$$
a_{L t} \sim a_{t}, \quad T\left(a_{L t}\right) \sim T\left(a_{t}\right) .
$$

(ii) [5] For $t>0$,

$$
Q\left(a_{t}\right) \sim \frac{t}{\sqrt{T\left(a_{t}\right)}}
$$

(iii) Let $\Lambda>1$ be defined in Definition 2.1 (d). Then we have

$$
a_{n} \leqslant C n^{1 / \Lambda} \text {. }
$$

Proof. We show (iii). From the definition of $T(x)$ we see

$$
|x|^{\Lambda} \leqslant C Q(x) .
$$

Hence, noting (ii) in this lemma, we have

$$
a_{n}^{\Lambda} \leqslant C Q\left(a_{n}\right) \leqslant C n .
$$

Therefore, we conclude (iii).\#

Lemma 3.2 [7]. Let $w \in \mathcal{F}_{\lambda}\left(C^{3}+\right)(0<\lambda<3 / 2)$. Let $1 \leqslant p \leqslant \infty$, and let $P \in \mathcal{P}_{n}$. Then we have

$$
\left\|\frac{w}{\sqrt{T}} P^{\prime}\right\|_{L_{p}(\mathbb{R})} \leqslant C \frac{n}{a_{n}}\|w P\|_{L_{p}(\mathbb{R})} .
$$

Moreover,

$$
\left\|w P^{\prime}\right\|_{L_{p}(\mathbb{R})} \leqslant C \frac{n}{a_{n}}\|\sqrt{T} w P\|_{L_{p}(\mathbb{R})} .
$$

Therefore, if $w \in \mathcal{F}_{\lambda}\left(C^{r+2}+\right)(0<\lambda<(r+2) /(r+1)), 1 \leqslant j \leqslant r$, where $r \geqslant 1$ is an integer,

$$
\left\|w P^{(j)}\right\|_{L_{p}(\mathbb{R})} \leqslant C\left(\frac{n}{a_{n}}\right)^{j}\left\|(\sqrt{T})^{j} w P\right\|_{L_{p}(\mathbb{R})}, \quad j=1,2,3, . ., r .
$$

We consider the connections of degree of approximation of a function between different norms. Levin and Lubinsky obtained a Nikolskii-type inequality as follows. 
Theorem 3.3 [5]. Let $w=\exp (-Q(x)) \in \mathcal{F}\left(C^{2}+\right)$, and let $P \in \mathcal{P}_{n}$. When $0<p \leqslant q \leqslant \infty$, we have

$$
\|w P\|_{L_{p}(\mathbb{R})} \leqslant C a_{n}^{\frac{1}{p}-\frac{1}{q}}\|w P\|_{L_{q}(\mathbb{R})},
$$

and when $0<q \leqslant p \leqslant \infty$, we have

$$
\|w P\|_{L_{p}(\mathbb{R})} \leqslant C\left(\frac{n \sqrt{T\left(a_{n}\right)}}{a_{n}}\right)^{\frac{1}{q}-\frac{1}{p}}\|w P\|_{L_{q}(\mathbb{R})} .
$$

We can obtain an analogy of Theorem 3.3 for the weight $w=\exp (-Q(x)) \in \mathcal{F}_{\lambda}\left(C^{3}+\right)(0<\lambda<3 / 2)$.

Theorem 3.4. Let $w=\exp (-Q) \in \mathcal{F}_{\lambda}\left(C^{3}+\right)(0<\lambda<3 / 2)$, and let $P \in \mathcal{P}_{n}$. For $0<p \leqslant q \leqslant \infty$, we have

$$
\|w P\|_{L_{p}(\mathbb{R})} \leqslant C a_{n}^{\frac{1}{p}-\frac{1}{q}}\|w P\|_{L_{q}(\mathbb{R})},
$$

and for $1 \leqslant q<p \leqslant \infty$, we have

$$
\|w P\|_{L_{p}(\mathbb{R})} \leqslant C\left(\frac{n}{a_{n}}\right)^{\frac{1}{q}-\frac{1}{p}}\left\|(\sqrt{T})^{\frac{1}{q}-\frac{1}{p}} w P\right\|_{L_{q}(\mathbb{R})} .
$$

To prove Theorem 3.4 we need some lemmas. We define

$$
\varphi_{u}(x)= \begin{cases}\frac{a_{u}}{u} \frac{1-\frac{|x|}{a_{u}}}{\sqrt{1-\frac{|x|}{a_{u}}+\delta_{u}}}, & |x| \leqslant a_{u} ; \quad \delta_{u}=\left\{u T\left(a_{u}\right)\right\}^{-2 / 3}, u>0 . \\ \varphi_{u}\left(a_{u}\right), & a_{u}<|x|,\end{cases}
$$

Lemma $3.5[7]$. We have

$$
\frac{a_{n}}{n} \frac{1}{\sqrt{T(x)}} \varphi_{n}^{-1}(x) \leqslant C
$$

We define $L_{p}$ Christoffel functions $\lambda_{n, p}(w ; x)$ by

$$
\lambda_{n, p}(w ; x):=\inf _{P \in \mathcal{P}_{n}} \int_{-\infty}^{\infty}|P w|^{p}(t) d t /|P(x)|^{p} .
$$

Lemma 3.6 [5]. Let $w \in \mathcal{F}\left(C^{2}+\right)$. Let $0<p<\infty$.

(i) Let $L>0$. Then uniformly for $n \geqslant 1$ and $|x| \leqslant a_{n}\left(1+L \eta_{n}\right)$, we have

$$
\lambda_{n, p}(w ; x) \sim \varphi_{n}(x) w^{p}(x) .
$$

(ii) Moreover, uniformly for $n \geqslant 1$ and $x \in \mathbb{R}$,

$$
\varphi_{n}(x) w^{p}(x) \leqslant C \lambda_{n, p}(w ; x) .
$$

Now the proof of Theorem 3.4 is simple.

Proof of Theorem 3.4. By Theorem 2.5 we can replace $(\sqrt{T})^{\frac{1}{q}-\frac{1}{p}} w$ with $w_{\alpha / 2} \in \mathcal{F}\left(C^{2}+\right)$, where $\alpha:=\frac{1}{q}-\frac{1}{p}$. The inequality (11) follows from the first inequality of Theorem 3.3. We show (12). Let $1 \leqslant q<p$.

$$
\begin{aligned}
& \|w P\|_{L_{p}(\mathbb{R})}^{p}=\int_{-\infty}^{\infty}|w(t) P(t)|^{p} d t=\int_{-\infty}^{\infty}|w(t) P(t)|^{p-q}|w(t) P(t)|^{q} d t=\int_{-\infty}^{\infty}\left|\frac{w(t) P(t)}{T^{\frac{\alpha}{2}} \frac{q}{p-q}(t)}\right|^{p-q}\left|w_{\frac{\alpha}{2}}(t) P(t)\right|^{q} d t \\
& \leqslant\left\|\frac{|w P|^{p}}{\sqrt{T}}\right\|_{L_{\infty}(\mathbf{R})}^{\frac{p-q}{p}}\left\|w_{\frac{\alpha}{2}} P\right\|_{L_{q}(\mathbb{R})}^{q},
\end{aligned}
$$


because of $\alpha p q /(p-q)=1$. Here we use $L_{p}$ Christoffel functions $\lambda_{n, p}(w ; x)$, and by Lemmas 3.5 and 3.6 we have

$$
\begin{aligned}
& \frac{|w(t) P(t)|^{p}}{\sqrt{T(t)}} \leqslant C \frac{w(t)^{p}}{\sqrt{T(t)}} \lambda_{n, p}^{-1}(w ; t)\|w P\|_{L_{p}(\mathbb{R})}^{p} \\
& \left.\leqslant C \frac{1}{\sqrt{T(t)}} \varphi_{n}^{-1}(t)\right)\|w P\|_{L_{p}(\mathbb{R})}^{p} \leqslant C\left(\frac{n}{a_{n}}\right)\|w P\|_{L_{p}(\mathbb{R})}^{p} .
\end{aligned}
$$

Substituting this estimate (14) into (13), we have

$$
\begin{aligned}
& \|w P\|_{L_{p}(\mathbb{R})}^{p} \leqslant C\left\{\left(\frac{n}{a_{n}}\right)\|w P\|_{L_{p}(\mathbb{R})}^{p}\right\}^{\frac{p-q}{p}}\left\|w_{\frac{\alpha}{2}} P\right\|_{L_{q}(\mathbb{R})}^{q} \\
& =C\left(\frac{n}{a_{n}}\right)^{\frac{p-q}{p}}\|w P\|_{L_{p}(\mathbb{R})}^{p-q}\left\|w_{\frac{\alpha}{2}} P\right\|_{L_{q}(\mathbb{R})}^{q} .
\end{aligned}
$$

So

$$
\|w P\|_{L_{p}(\mathbb{R})}^{q} \leqslant C\left(\frac{n}{a_{n}}\right)^{\frac{p-q}{p}}\left\|w_{\frac{\alpha}{2}} P\right\|_{L_{q}(\mathbb{R})}^{q}
$$

that is,

$$
\|w P\|_{L_{p}(\mathbb{R})} \leqslant C\left(\frac{n}{a_{n}}\right)^{\frac{1}{q}-\frac{1}{p}}\left\|w_{\frac{\alpha}{2}} P\right\|_{L_{q}(\mathbb{R})}
$$

consequently, we have the result (12). \#

The next lemma is useful.

Lemma 3.7. Let $\left\{b_{k}\right\}_{k=0}^{\infty}$ be an increasing sequence of positive numbers, and $\left\{c_{k}\right\}_{k=0}^{\infty}$ be a decreasing sequence of positive numbers. Let $j \geqslant 1$ be an integer. Then we have

$$
\sum_{k=0}^{j-1} 2^{k} b_{2^{k}} c_{2^{k+1}} \leqslant \sum_{i=1}^{2^{j}} b_{i} c_{i} \leqslant b_{1} c_{1}+\sum_{k=0}^{j-1} 2^{k} b_{2^{k+1}} c_{2^{k}}
$$

Proof.

$$
\begin{aligned}
& \sum_{i=1}^{2^{j}} b_{i} c_{i}=b_{1} c_{1}+\sum_{k=1}^{j} \sum_{i=2^{k-1}+1}^{2^{k}} b_{i} c_{i} \\
& \geqslant b_{1} c_{1}+\sum_{k=1}^{j} 2^{k-1} b_{2^{k-1}} c_{2^{k}} \geqslant \sum_{k=0}^{j-1} 2^{k} b_{2^{k}} c_{2^{k+1}},
\end{aligned}
$$

and

$$
\begin{aligned}
& \sum_{i=1}^{2^{j}} b_{i} c_{i}=b_{1} c_{1}+\sum_{k=1}^{j} \sum_{i=2^{k-1}+1}^{2^{k}} b_{i} c_{i} \\
& \leqslant b_{1} c_{1}+\sum_{k=1}^{j} 2^{k-1} b_{2^{k}} c_{2^{k-1}}=b_{1} c_{1}+\sum_{k=0}^{j-1} 2^{k} b_{2^{k+1}} c_{2^{k}} \cdot \#
\end{aligned}
$$

In the rest of the paper we write $f^{(s)}=g$ if $f$ is an $s$-times iterated integral of a function $g$ such that $w g \in L_{p}(\mathbb{R})$. 
Proof of Theorem 2.8. Let $p \leqslant q$. We write $\alpha:=1 / p-1 / q$. Let $n \geqslant s$ be fixed, and let $T^{\alpha / 2} w \sim w_{\alpha / 2} \in$ $\mathcal{F}\left(C^{2}+\right)$.

(i) We can find polynomials $P_{j} \in \mathcal{P}_{2^{j} n}$ such that for $j=0,1,2, \ldots$,

$$
\left\|w\left(f-P_{j}\right)\right\|_{L_{q}(\mathbb{R})} \leqslant\left\|w_{\left(\frac{1}{2} ; s\right)}\left(f-P_{j}\right)\right\|_{L_{q}(\mathbb{R})} \leqslant 2 E_{q, 2^{j} n}\left(w_{\left(\frac{1}{2} ; s\right)} ; f\right) .
$$

Since we have $\lim _{j \rightarrow \infty} E_{q, 2^{j} n}\left(w_{\left(\frac{1}{2} ; s\right)} ; f\right)=0$, for with $R_{j}:=P_{j+1}-P_{j}, j=0,1,2, \ldots$, we see

$$
f=P_{0}+\sum_{j=0}^{\infty} R_{j}
$$

in the sense of

$$
\lim _{m \rightarrow \infty}\left\|w_{\left(\frac{1}{2} ; s\right)}\left(f-\left(P_{0}+\sum_{j=0}^{m-1} R_{j}\right)\right)\right\|_{L_{q}(\mathbb{R})}=\lim _{m \rightarrow \infty}\left\|w_{\left(\frac{1}{2} ; s\right)}\left(f-P_{m}\right)\right\|_{L_{q}(\mathbb{R})}=0 .
$$

Using the Nikolskii-type inequality (11) and the Markov-Bernstein-type inequality (Lemma 3.2), we get

$$
\begin{aligned}
& \left\|w P_{0}^{(s)}\right\|_{L_{p}(\mathbb{R})}+\sum_{j=0}^{\infty}\left\|w R_{j}^{(s)}\right\|_{L_{p}(\mathbb{R})} \leqslant C\left[1+\sum_{j=0}^{\infty} a_{2^{j+1} n}^{\alpha}\left\|w R_{j}^{(s)}\right\|_{L_{q}(\mathbb{R})}\right] \\
& \leqslant C\left[1+\sum_{j=0}^{\infty} a_{2^{j+1} n}^{\alpha}\left(\frac{2^{j+1} n}{a_{2^{j+1} n}}\right)^{s}\left\|w_{\left(\frac{1}{2} ; s\right)} R_{j}\right\|_{L_{q}(\mathbb{R})}\right] \\
& \leqslant C\left[1+\sum_{j=0}^{\infty} a_{2^{j+1} n}^{\alpha}\left(\frac{2^{j+1} n}{a_{2^{j+1} n}}\right)^{s}\left\|w_{\left(\frac{1}{2} ; s\right)}\left(P_{j+1}-f+f-P_{j}\right)\right\|_{L_{q}(\mathbb{R})}\right] \\
& \leqslant C\left[1+\sum_{j=1}^{\infty} a_{2^{j} n}^{\alpha}\left(\frac{2^{j} n}{a_{2^{j} n}}\right)^{s} E_{q, 2^{j} n}\left(w_{\left(\frac{1}{2} ; s\right)} ; f\right)\right] .
\end{aligned}
$$

Here by (3) we will see that the last sum is finite. Then, there is a function $f^{(s)} \in L_{p, w}(\mathbb{R})$ such that

$$
f^{(s)}=P_{0}^{(s)}+\sum_{j=0}^{\infty} R_{j}^{(s)}
$$

in the sense of $L_{p, w}(\mathbb{R})$ convergence. Now, we show that the last sum (15) is finite. We use the first inequality in Lemma 3.7 with

$$
b_{i}=a_{i n}^{\alpha-s}(i n)^{s}, \quad c_{i}=\frac{E_{q, i n}\left(w_{\left(\frac{1}{2} ; s\right)} ; f\right)}{i} . \quad i=1,2,3, \ldots .
$$

Then, in (15),

$$
\begin{aligned}
& \sum_{j=1}^{\infty} a_{2^{j} n}^{\alpha}\left(\frac{2^{j} n}{a_{2^{j} n}}\right)^{s} E_{q, 2^{j} n}\left(w_{\left(\frac{1}{2} ; s\right)} ; f\right) \leqslant 2^{s+1} \sum_{j=1}^{\infty} a_{2^{j-1} n}^{\alpha-s}\left(2^{j-1} n\right)^{s} E_{q, 2^{j} n}\left(w_{\left(\frac{1}{2} ; s\right)} ; f\right) \\
& =2^{s+1} \sum_{j=1}^{\infty} 2^{j-1} b_{2^{j-1}} c_{2^{j}} \leqslant 2^{s+2} \sum_{i=1}^{\infty} b_{i} c_{i} \leqslant 2^{s+2} \sum_{j=1}^{\infty} a_{j n}^{\alpha-s}(j n)^{s} \frac{E_{q, j n}\left(w_{\left(\frac{1}{2} ; s\right)} ; f\right)}{j} .
\end{aligned}
$$

So,

$$
\begin{aligned}
& E_{p, n-s}\left(w ; f^{(s)}\right) \leqslant\left\|w\left(f^{(s)}-P_{0}^{(s)}\right)\right\|_{L_{p}(\mathbf{R})} \\
& \leqslant \sum_{j=0}^{\infty}\left\|w R_{j}^{(s)}\right\|_{L_{p}(\mathbb{R})} \leqslant C \sum_{k=1}^{\infty} a_{k n}^{\alpha-s}(k n)^{s} \frac{E_{q, k n}\left(w_{\left(\frac{1}{2} ; s\right)} ; f\right)}{k},
\end{aligned}
$$


that is, (17) means (4). Now we will show

$$
\sum_{k=1}^{\infty} a_{k n}^{\alpha-s}(k n)^{s} \frac{E_{q, k n}\left(w_{\left(\frac{1}{2} ; s\right)} ; f\right)}{k}<\infty .
$$

We see

$$
\begin{aligned}
& \sum_{k=1}^{\infty} a_{k n}^{\alpha-s}(k n)^{s} \frac{E_{q, k n}\left(w_{\left(\frac{1}{2} ; s\right)} ; f\right)}{k}=\sum_{k=1}^{\infty} n a_{k n}^{\alpha-s}(k n)^{s} \frac{E_{q, k n}\left(w_{\left(\frac{1}{2} ; s\right)} ; f\right)}{k n} \\
& =n a_{n}^{\alpha-s} n^{s} \frac{E_{q, n}\left(w_{\left(\frac{1}{2} ; s\right)} ; f\right)}{n}+\sum_{k=2}^{\infty} n a_{k n}^{\alpha-s}(k n)^{s} \frac{E_{q, k n}\left(w_{\left(\frac{1}{2} ; s\right)} ; f\right)}{k n} .
\end{aligned}
$$

Let $0 \leqslant j \leqslant[(n+1) / 2]([x]$ is the largest integer $\leqslant x)$. Since $a_{n+j}^{\alpha-s}(n+j)^{s} \sim a_{n-j}^{\alpha-s}(n-j)^{s}$ uniformly for $0 \leqslant j \leqslant[(n+1) / 2]$ (see Lemma 3.1 (i)), we have

$$
\begin{aligned}
& n a_{n}^{\alpha-s} n^{s} \frac{E_{q, n}\left(w_{\left(\frac{1}{2} ; s\right)} ; f\right)}{n} \leqslant 2 \sum_{j=0}^{[(n+1) / 2]} a_{n+j}^{\alpha-s}(n+j)^{s} \frac{E_{q, n-j}\left(w_{\left(\frac{1}{2} ; s\right)} ; f\right)}{n-j} \\
& \leqslant C \sum_{j=0}^{[(n+1) / 2]} a_{n-j}^{\alpha-s}(n-j)^{s} \frac{E_{q, n-j}\left(w_{\left(\frac{1}{2} ; s\right)} ; f\right)}{n-j} \\
& \leqslant C \sum_{j=1}^{n} a_{j}^{\alpha-s} j^{s} \frac{E_{q, j}\left(w_{\left(\frac{1}{2} ; s\right)} ; f\right)}{j} .
\end{aligned}
$$

Similarly, for $k \geqslant 2$ we also have

$$
\begin{aligned}
& n a_{k n}^{\alpha-s}(k n)^{s} \frac{E_{q, k n}\left(w_{\left(\frac{1}{2} ; s\right)} ; f\right)}{k n} \\
& \leqslant C \sum_{j=1}^{n} a_{(k-1) n+j}^{\alpha-s}((k-1) n+j)^{s} \frac{E_{q,(k-1) n+j}\left(w_{\left(\frac{1}{2} ; s\right)} ; f\right)}{(k-1) n+j} .
\end{aligned}
$$

So we have

$$
\begin{aligned}
& \sum_{k=2}^{\infty} n a_{k n}^{\alpha-s}(k n)^{s} \frac{E_{q, k n}\left(w_{\left(\frac{1}{2} ; s\right)} ; f\right)}{k n} \\
& \leqslant C \sum_{k=2}^{\infty}\left\{\sum_{j=1}^{n} a_{(k-1) n+j}^{\alpha-s}((k-1) n+j)^{s} \frac{E_{q,(k-1) n+j}\left(w_{\left(\frac{1}{2} ; s\right)} ; f\right)}{(k-1) n+j}\right\} \\
& \leqslant C \sum_{j=1}^{\infty} a_{n+j}^{\alpha-s}(n+j)^{s} \frac{E_{q, n+j}\left(w_{\left(\frac{1}{2} ; s\right)} ; f\right)}{n+j}
\end{aligned}
$$

By (19), (20) and the assumption (3) we conclude (18) as follows:

$$
\sum_{k=1}^{\infty} n a_{k n}^{\alpha-s}(k n)^{s} \frac{E_{q, k n}\left(w_{\left(\frac{1}{2} ; s\right)} ; f\right)}{k n} \leqslant C \sum_{j=1}^{\infty} a_{j}^{\alpha-s} j^{s-1} E_{q, j}\left(w_{\left(\frac{1}{2} ; s\right)} ; f\right)<\infty,
$$

that is, we see that $f$ is an $s$-times iterated integral of a function $f^{(s)}$ almost everywhere. Consequently, noting $w_{\left(\frac{1}{2} ; s\right)} \sim T^{\frac{s}{2}} w$, we have (4).

(ii) Let $\beta=1 / q-1 / p>0$. We have (6) as above. In fact, let $n \geqslant s$ be fixed. We can find polynomials $P_{j} \in \mathcal{P}_{2^{j} n}$ such that for $j=0,1,2, \ldots$,

$$
\begin{aligned}
& \left\|w\left(f-P_{j}\right)\right\|_{L_{q}(\mathbb{R})} \leqslant\left\|\left\{w_{\frac{\beta}{2}}\right\}_{\left(\frac{1}{2} ; s\right)}\left(f-P_{j}\right)\right\|_{L_{q}(\mathbb{R})} \\
& \leqslant 2 E_{q, 2^{j} n}\left(\left\{w_{\frac{\beta}{2}}\right\}_{\left(\frac{1}{2} ; s\right)} ; f\right) .
\end{aligned}
$$


From $\lim _{j \rightarrow \infty} E_{q, 2^{j} n}\left(\left\{w_{\frac{\beta}{2}}\right\}_{\left(\frac{1}{2} ; s\right)} ; f\right)=0$, we have

$$
f=P_{0}+\sum_{j=0}^{\infty} R_{j}, \quad \text { where } R_{j}:=P_{j+1}-P_{j},
$$

in the sense of $L_{q,\left\{w_{\frac{\beta}{2}}\right\}_{\left(\frac{1}{2} ; s\right)}}(\mathbb{R})$-convergence, and

$$
\left\|\left\{w_{\frac{\beta}{2}}\right\}_{\left(\frac{1}{2} ; s\right)} R_{j}\right\|_{L_{p}(\mathbb{R})} \leqslant 4 E_{p, 2^{j} n}\left(\left\{w_{\frac{\beta}{2}}\right\}_{\left(\frac{1}{2} ; s\right)} ; f\right), \quad j=0,1,2, \ldots .
$$

Now, by Nikolskii-type inequality (12) and Lemma 3.2 we have for some constant $C>0$,

$$
\begin{aligned}
& \left\|w P_{0}^{(s)}\right\|_{L_{p}(\mathbb{R})}+\sum_{j=0}^{\infty}\left\|w R_{j}^{(s)}\right\|_{L_{p}(\mathbb{R})} \leqslant C+\sum_{j=0}^{\infty}\left(\frac{2^{j} n}{a_{2^{j} n}}\right)^{\beta}\left\|\left\{w_{\frac{\beta}{2}}\right\}_{\left(\frac{1}{2} ; s\right)} R_{j}^{(s)}\right\|_{L_{q}(\mathbb{R})} \\
& \leqslant C\left[1+\sum_{j=0}^{\infty}\left(\frac{2^{j} n}{a_{2^{j} n}}\right)^{\beta}\left(\frac{2^{j} n}{a_{2^{j} n}}\right)^{s}\left\|\left\{w_{\frac{\beta}{2}}\right\}_{\left(\frac{1}{2} ; s\right)} R_{j}\right\|_{L_{q}(\mathbb{R})}\right] \\
& \leqslant C\left[1+\sum_{j=0}^{\infty}\left(\frac{2^{j} n}{a_{2^{j} n}}\right)^{\beta+s} E_{q, 2^{j} n}\left(\left\{w_{\frac{\beta}{2}}\right\}_{\left(\frac{1}{2} ; s\right)} ; f\right)\right] \\
& \leqslant C\left[1+\left(\frac{n}{a_{n}}\right)^{\beta+s} \frac{E_{q, n}\left(\left\{w_{\frac{\beta}{2}}\right\}_{\left(\frac{1}{2} ; s\right)} ; f\right)}{2^{j-1}}+2^{\beta+s+1} \sum_{j=1}^{\infty} 2^{j-1}\left(\frac{2^{j-1} n}{a_{2^{j-1} n}}\right)^{\beta+s} \frac{E_{q, 2^{j} n}\left(\left\{w_{\frac{\beta}{2}}\right\}_{\left(\frac{1}{2} ; s\right)} ; f\right)}{2^{j}}\right] .
\end{aligned}
$$

Using Lemma 3.7 with $b_{j-1}=\left(\frac{2^{j-1} n}{a_{2^{j-1}}}\right)^{\beta+s}, c_{j}=\frac{E_{q, 2_{n} j_{n}}\left(\left\{w_{\frac{\beta}{2}}\right\}_{\left(\frac{1}{2} ; s\right)} ; f\right)}{2^{j}}$, we obtain

$$
\leqslant C\left[1+\sum_{k=1}^{\infty}\left(\frac{k n}{a_{k n}}\right)^{\beta+s} \frac{E_{q, k n}\left(\left\{w_{\frac{\beta}{2}}\right\}_{\left(\frac{1}{2} ; s\right)} ; f\right)}{k}\right] .
$$

Furthermore,

$$
\begin{aligned}
& \left(\frac{[(n+1) / 2]}{a_{[(n+1) / 2]}}\right)^{\beta+s} \frac{E_{q,[(n+1) / 2]}\left(\left\{w_{\frac{\beta}{2}}\right\}_{\left(\frac{1}{2} ; s\right)} ; f\right)}{[(n+1) / 2]}+\left(\frac{[(n+1) / 2]+1}{a_{[(n+1) / 2]+1}}\right)^{\beta+s} \frac{E_{q, n / 2+2}\left(\left\{w_{\frac{\beta}{2}}\right\}_{\left(\frac{1}{2} ; s\right)} ; f\right)}{[(n+1) / 2]+1} \\
& +\ldots .+\left(\frac{n}{a_{n}}\right)^{\beta+s} \frac{E_{q, n}\left(\left\{w_{\frac{\beta}{2}}\right\}_{\left(\frac{1}{2} ; s\right)} ; f\right)}{n} \\
& \geqslant C \frac{n}{2}\left(\frac{n}{a_{n}}\right)^{\beta+s} \frac{E_{q, n}\left(\left\{w_{\frac{\beta}{2}}\right\}_{\left(\frac{1}{2} ; s\right)} ; f\right)}{n} \text { (by } \frac{[(n+1) / 2]}{a_{[(n+1) / 2]}} \sim \frac{n}{a_{n}}(\text { see Lemma 3.1 (i) }) \\
& \geqslant C\left(\frac{n}{a_{n}}\right)^{\beta+s} E_{q, n}\left(\left\{w_{\frac{\beta}{2}}\right\}_{\left(\frac{1}{2} ; s\right)} ; f\right) .
\end{aligned}
$$

Similarly, for $k \geqslant 2$

$$
\begin{aligned}
& \left(\frac{(k-1) n+1}{a_{(k-1) n+1}}\right)^{\beta+s} \frac{E_{q,(k-1) n+1}\left(\left\{w_{\frac{\beta}{2}}\right\}_{\left(\frac{1}{2} ; s\right)} ; f\right)}{(k-1) n+1} \\
& +\left(\frac{(k-1) n+2}{a_{(k-1) n+2}}\right)^{\beta+s} \frac{E_{q,(k-1) n+2}\left(\left\{w_{\frac{\beta}{2}}\right\}_{\left(\frac{1}{2} ; s\right)} ; f\right)}{(k-1) n+1} \\
& +\ldots .+\left(\frac{k n}{a_{k n}}\right)^{\beta+s} \frac{E_{q, k n}\left(\left\{w_{\frac{\beta}{2}}\right\}_{\left(\frac{1}{2} ; s\right)} ; f\right)}{k n} \\
& \geqslant C n\left(\frac{k n}{a_{k n}}\right)^{\beta+s} \frac{E_{q, k n}\left(\left\{w_{\frac{\beta}{2}}\right\}_{\left(\frac{1}{2} ; s\right)} ; f\right)}{k n} \\
& \geqslant C\left(\frac{k n}{a_{k n}}\right)^{\beta+s} \frac{E_{q, k n}\left(\left\{w_{\frac{\beta}{2}}\right\}_{\left(\frac{1}{2} ; s\right)} ; f\right)}{k} .
\end{aligned}
$$


Therefore, by the assumption (5),

$$
\begin{aligned}
& C\left[1+\sum_{k=1}^{\infty}\left(\frac{k n}{a_{k n}}\right)^{\beta+s} \frac{E_{q, k n}\left(\left\{w_{\frac{\beta}{2}}\right\}_{\left(\frac{1}{2} ; s\right)} ; f\right)}{k}\right] \\
& \leqslant C\left[1+\sum_{n=1}^{\infty}\left(\frac{n}{a_{n}}\right)^{\beta+s} \frac{E_{q, n}\left(\left\{w_{\frac{\beta}{2}}\right\}_{\left(\frac{1}{2} ; s\right)} ; f\right)}{n}\right]<\infty .
\end{aligned}
$$

So, from $(21)$ we see that $f$ is an $s$-times iterated integral of a function $f^{(s)}$ almost everywhere. Moreover, from the above estimation (21) we have

$$
\begin{aligned}
& E_{p, n-s}\left(w ; f^{(s)}\right) \leqslant\left\|w\left(f^{(s)}-P_{0}^{(s)}\right)\right\|_{L_{p}(\mathbb{R})} \leqslant \sum_{j=0}^{\infty}\left\|w R_{j}^{(s)}\right\|_{L_{p}(\mathbb{R})} \\
& \leqslant C \sum_{k=1}^{\infty}\left(\frac{k n}{a_{k n}}\right)^{\beta+s} \frac{E_{q, k n}\left(\left\{w_{\frac{\beta}{2}}\right\}_{\left(\frac{1}{2} ; s\right)} ; f\right)}{k} .
\end{aligned}
$$

Therefore, noting $\left\{w_{\frac{\beta}{2}}\right\}_{\left(\frac{1}{2} ; s\right)} \sim T^{\left(s+\left(\frac{1}{p}-\frac{1}{q}\right)\right) / 2} w$, we have $(6)$.

Next, we let $p \leqslant q$, and put $\alpha=1 / p-1 / q$. If $T(x)$ is unbounded, then from Remark 1.9 we find that for any fixed $d>0$ there exist $C(\delta)>0$ and $N(d)>0$ such that

$$
a_{n}^{d} \leqslant C(\delta) n \text { for } n \geqslant N(\delta)
$$

If $T(x)$ is bounded, then from our assumption we have (22) with $d=2$. Therefore, the condition of (5) means (3), because

$$
a_{n}^{\alpha-s} n^{s}=\frac{a_{n}^{2 \alpha}}{n^{\alpha}}\left(\frac{n}{a_{n}}\right)^{\alpha+s} \leqslant C\left(\frac{n}{a_{n}}\right)^{\alpha+s}
$$

Therefore, applying (i) above with the weight $w, f$ is an $s$-times iterated integral of a function $f^{(s)} \in$ $L_{p, w}(\mathbb{R})$, and we have for the integer $n \geqslant s$,

$$
\begin{aligned}
& E_{p, n-s}\left(w ; f^{(s)}\right) \leqslant C \sum_{k=1}^{\infty} a_{k n}^{\alpha-s}(k n)^{s} \frac{E_{q, k n}\left(\left\{w_{\frac{\alpha}{2}}\right\}_{\left(\frac{1}{2} ; s\right)} ; f\right)}{k} \\
& \leqslant C \sum_{k=1}^{\infty}\left(\frac{k n}{a_{k n}}\right)^{\alpha+s} \frac{E_{q, k n}\left(\left\{w_{\frac{\alpha}{2}}\right\}_{\left(\frac{1}{2} ; s\right)} ; f\right)}{k}
\end{aligned}
$$

that is, noting $\left\{w_{\frac{\alpha}{2}}\right\}_{\left(\frac{1}{2} ; s\right)} \sim T^{\left(s+\left(\frac{1}{p}-\frac{1}{q}\right)\right) / 2} w$, we have the result.\#

\section{Proof of Corollaries 2.10 and 2.11}

In this section we prove Corollary 2.10 and Corollary 2.11.

Proof of Corollary 2.10. (i) We use Theorem 2.8 (i) with $p=q$. We assume (7). Then, by Lemma 3.1 (iii) we see

$$
\begin{aligned}
& \sum_{n=1}^{\infty} a_{k}^{-s} k^{s-1} E_{p, k}\left(w_{\left(\frac{1}{2} ; s\right)} ; f\right) \leqslant \sum_{k=1}^{\infty}\left(\frac{k}{a_{k}}\right)^{s}\left(\frac{a_{k}}{k}\right)^{\beta}\left(\frac{1}{k}\right) \leqslant \sum_{k=1}^{\infty}\left(\frac{a_{k}}{k}\right)^{\beta-s}\left(\frac{1}{k}\right) \\
& \leqslant \sum_{k=1}^{\infty}\left(\frac{1}{k}\right)^{1+\left(1-\frac{1}{\Lambda}\right)(\beta-s)}<\infty
\end{aligned}
$$


that is, (3) is satisfied. Therefore, $f$ is an s-times iterated integral of a function $f^{(s)} \in L_{p, w}(\mathbb{R})$, and then we have (4), that is,

$$
\begin{aligned}
& E_{p, n-s}\left(w ; f^{(s)}\right) \leqslant C \sum_{k=1}^{\infty}\left(\frac{k n}{a_{k n}}\right)^{s} \frac{E_{p, k n}\left(w_{\left(\frac{1}{2} ; s\right)} ; f\right)}{k} \leqslant C \sum_{k=1}^{\infty}\left(\frac{a_{k n}}{k n}\right)^{\beta-s} \frac{1}{k} \\
& \leqslant C \int_{1}^{\infty}\left(\frac{a_{t n}}{t n}\right)^{\beta-s} \frac{1}{t} d t \leqslant C \int_{n}^{\infty}\left(\frac{a_{u}}{u}\right)^{\beta-s} \frac{1}{u} d u=C \int_{n}^{\infty} u^{s-\beta-1} a_{u}^{\beta-s} d u .
\end{aligned}
$$

Now, by [5] we see that

$$
\frac{a_{t}^{\prime}}{a_{t}} \leqslant C \frac{1}{T\left(a_{t}\right) t}
$$

Hence,

$$
\begin{aligned}
& A:=\int_{n}^{\infty} u^{s-\beta-1} a_{u}^{\beta-s} d u=\frac{1}{s-\beta}\left[\left.u^{s-\beta} a_{u}^{\beta-s}\right|_{n} ^{\infty}-\int_{n}^{\infty} u^{s-\beta} a_{u}^{\beta-s-1} a_{u}{ }^{\prime} d u\right] \\
& \leqslant C\left(\frac{a_{n}}{n}\right)^{\beta-s}+\frac{1}{T\left(a_{n}\right)} \int_{n}^{\infty} u^{s-\beta-1} a_{u}^{\beta-s} d u \leqslant C\left(\frac{a_{n}}{n}\right)^{\beta-s}+\frac{A}{T\left(a_{n}\right)} .
\end{aligned}
$$

Therefore, we have

$$
E_{p, n-s}\left(w ; f^{(s)}\right) \leqslant C A \leqslant C\left(\frac{a_{n}}{n}\right)^{\beta-s} .
$$

Here we replace $n-s$ with $n$, then we have the result because of $a_{n+s} /(n+s) \sim a_{n} / n$.

(ii) Let $1 \leqslant p, q \leqslant \infty$. We use Theorem 2.8 (ii). Let us assume (8). Then

$$
\begin{aligned}
& \sum_{n=1}^{\infty}\left(\frac{n}{a_{n}}\right)^{s+|\alpha|} \frac{E_{q, n}\left(\left\{w_{\frac{|\alpha|}{2}}\right\}_{\left(\frac{1}{2} ; s\right)} ; f\right)}{n} \leqslant C \sum_{n=1}^{\infty}\left(\frac{n}{a_{n}}\right)^{s+|\alpha|}\left(\frac{a_{n}}{n}\right)^{\beta} \frac{1}{n} \\
& \leqslant C \sum_{n=1}^{\infty}\left(\frac{a_{n}}{n}\right)^{\beta-s-|\alpha|} \frac{1}{n} \leqslant C \sum_{n=1}^{\infty}\left(\frac{1}{n}\right)^{1+\left(1-\frac{1}{\Lambda}\right)(\beta-s-|\alpha|)}<\infty,
\end{aligned}
$$

so, (5) is satisfied. Therefore, $f$ is an $s$-times iterated integral of a function $f^{(s)} \in L_{p, w}(\mathbb{R})$, and from $(7)$ we have

$$
E_{p, n}\left(w ; f^{(s)}\right) \leqslant C \sum_{k=1}^{\infty}\left(\frac{k n}{a_{k n}}\right)^{s+|\alpha|} \frac{E_{q, k n}\left(\left\{w_{\frac{|\alpha|}{2}}\right\}_{\left(\frac{1}{2} ; s\right)} ; f\right)}{k} \leqslant C \sum_{k=1}^{\infty}\left(\frac{a_{k n}}{k n}\right)^{\beta-s-|\alpha|} \frac{1}{k} .
$$

Here, as the proof of (i), we have

$$
E_{p, n}\left(w ; f^{(s)}\right)=\mathcal{O}\left(\frac{a_{n}}{n}\right)^{\beta-s-|\alpha|} . \quad \#
$$

To prove Corollary 2.11 we need a lemma;

Lemma 4.1 ([5]). For the weight $w_{l ; \gamma}(x)=\exp _{l}\left(|x|^{\gamma}\right)-\exp _{l}(0), \quad \gamma>1$, and $l \geqslant 1$ is an integer. We know

$$
a_{n}=\left(\log _{l}(n+1)\right)^{\frac{1}{\gamma}}(1+o(1)), n=1,2,3, \ldots
$$

and for some $c$ and $C>0$,

$$
T\left(a_{n}\right)\left\{\begin{array}{lr}
\sim \log (n+1) & \text { if } l=1 \\
\geqslant C\left\{\log _{l}(n+1)\right\}\left(\log _{l-1}(n+1)\right)^{c} & \text { if } l \geqslant 2
\end{array}\right.
$$

where $\log _{0} x=1$. 
Proof of Corollary 2.11. We use Theorem 2.8 (i). As we see in the proof of Corollary 2.10 (i), we have (3). Therefore, by (4), (9) and (23),

$$
\begin{aligned}
& E_{p, n-s}\left(w_{l ; \gamma} ; f\right) \leqslant C \sum_{k=1}^{\infty} a_{k n}^{\frac{1}{p}-\frac{1}{q}-s}(k n)^{s} \frac{E_{q, k n}\left(T^{\frac{s}{2}} w_{l ; \gamma} ; f\right)}{k} \\
& \leqslant C \sum_{k=1}^{\infty} a_{k n}^{\frac{1}{p}-\frac{1}{q}-s}(k n)^{s}\left(\frac{a_{k n}}{k n}\right)^{\beta} \frac{1}{k} \leqslant C \int_{n}^{\infty} t^{s-\beta-1} a_{t}^{\beta-s+\frac{1}{p}-\frac{1}{q}} d t \\
& \leqslant\left. C \frac{-1}{\beta-s} t^{s-\beta} a_{t}^{\beta-s+\frac{1}{p}-\frac{1}{q}}\right|_{n} ^{\infty}+\frac{\beta-s+\frac{1}{p}-\frac{1}{q}}{\beta-s} \int_{n}^{\infty} t^{s-\beta} a_{t}^{\beta-s+\frac{1}{p}-\frac{1}{q}-1} a_{t}^{\prime} d t \\
& \leqslant C \frac{1}{\beta-s} n^{s-\beta} a_{n}^{\beta-s+\frac{1}{p}-\frac{1}{q}}+\frac{\beta-s+\frac{1}{p}-\frac{1}{q}}{\beta-s} \int_{n}^{\infty} t^{s-\beta-1} \frac{a_{t}^{\beta-s+\frac{1}{p}-\frac{1}{q}}}{T\left(a_{t}\right)} d t .
\end{aligned}
$$

We will estimate the last term in $(24)$. Then we use $a_{t} \sim\left(\log _{l} t\right)^{1 / \gamma}$. By Lemma 3.4, if $l>1$, then we have

$$
\int_{n}^{\infty} t^{s-\beta-1} \frac{a_{t}^{\beta-s+\frac{1}{p}-\frac{1}{q}}}{T\left(a_{t}\right)} d t \leqslant \int_{n}^{\infty} t^{s-\beta-1} \frac{\left\{\log _{l} t\right\}^{\left(\beta-s+\frac{1}{p}-\frac{1}{q}\right) / \gamma}}{C\left\{\log _{l} t\right\}\left(\log _{l-1} t\right)^{c}} d t \leqslant C \int_{n}^{\infty} t^{s-\beta-1} d t \leqslant C n^{s-\beta}
$$

by $\left(\log _{l} t\right)^{\nu} \leqslant C \log _{l-1} t$ for $\nu \in \mathbb{R}$ and $t$ large enough. Let $l=1$. If $\beta-s+\frac{1}{p}-\frac{1}{q} \leqslant \gamma$, then we also see

$$
\int_{n}^{\infty} t^{s-\beta-1} \frac{a_{t}^{\beta-s+\frac{1}{p}-\frac{1}{q}}}{T\left(a_{t}\right)} d t \leqslant C \int_{n}^{\infty} t^{s-\beta-1} d t \leqslant C n^{s-\beta}
$$

If $l=1$ and $\beta-s+\frac{1}{p}-\frac{1}{q}>\gamma$, then we fix any $\delta ; 0<\delta<\beta-s$. Then, noting Lemma 4.1, we have

$$
\int_{n}^{\infty} t^{s-\beta-1} \frac{a_{t}^{\beta-s+\frac{1}{p}-\frac{1}{q}}}{T\left(a_{t}\right)} d t \leqslant C \int_{n}^{\infty} t^{s-\beta+\delta-1} d t \leqslant C n^{s-\beta+\delta} .
$$

Then, from (25)-(27), we have

$$
\begin{aligned}
& E_{p, n}\left(w_{l ; \gamma} ; f^{(s)}\right) \leqslant C n^{s-\beta} a_{n}^{\beta-s+\frac{1}{p}-\frac{1}{q}} \\
& =\left\{\begin{array}{l}
\mathcal{O}\left(n^{-\beta+s+\delta}\right), \quad \text { where } 0<\delta<\beta-s, \text { for } l=1 \text { and } \beta-s+\frac{1}{p}-\frac{1}{q}>\gamma \\
\mathcal{O}\left(n^{-\beta+s}\left(\log _{l} n\right)^{\frac{1}{\gamma}\left(\beta-s-\frac{1}{q}+\frac{1}{p}\right)}\right), \text { otherwise, }
\end{array}\right.
\end{aligned}
$$

that is, we conclude the result (10).\#

\section{References}

1. H. N. Mhaskar, "Introduction to the Theory of Weighted Polynomial Approximation," World Scientific, Singapore, 1996.

2. G. G. Lorentz, "Approximation of Functions", Holt, Rinehart and Winston, 1966.

3. S. B. Damelin, "Converse and Smoothness Theorems for Erd"os Weights in Lp $(0<\mathrm{p} 6 \infty)$,"Journal of Approximation Theory 93 (1998), 349-398.

4. S. B. Damelin and D. S. Lubinsky, "Jackson Theorems for Erd" os Weights in Lp $(0<\mathrm{p} 6 \infty)$,"Journal of Approximation Theory 94 (1998), 333-382.

5. A. L. Levin and D. S. Lubinsky, "Orthogonal Polynomials for Exponential Weights," Springer, New York, 2001.

6. H. S. Jung and R. Sakai, "Specific examples of exponential weights, Commun." Korean Math. Soc. 24 (2009), No.2, 303-319.

7. R. Sakai and N. Suzuki, "Mollification of exponential weights and its application to the Markov-Bernstein inequality," Pioneer J. of Math., Vol.7, no.1 (2013) 83-101.

8. R. Sakai and N. Suzuki, "Favard-type inequalities for exponential weights," Pioneer J. of Math. vol 3. No.1 (2011), 1-16. 